\title{
NUMERICAL SOLUTIONS OF DIFFERENTIAL-ALGEBRAIC EQUATIONS AND ITS APPLICATIONS IN SOLVING TPPC PROBLEMS
}

\author{
He-Sheng Wang \\ Taiwan., hswang@mail.ntou.edu.tw \\ Wei-Lun Jhu \\ Department of Electrical Engineering, National Taiwan Ocean University, Keelung, Taiwan. \\ Chee-Fai Yung \\ Department of Electrical Engineering, National Taiwan Ocean University, Keelung, Taiwan. \\ Ping-Feng Wang \\ Institute for Information Industry, Taipei, Taiwan.
}

Department of Communications, Navigation and Control Engineering National Taiwan Ocean University, Keelung 202,

Follow this and additional works at: https://jmstt.ntou.edu.tw/journal

Part of the Computer Sciences Commons, and the Engineering Commons

\author{
Recommended Citation \\ Wang, He-Sheng; Jhu, Wei-Lun; Yung, Chee-Fai; and Wang, Ping-Feng (2011) "NUMERICAL SOLUTIONS OF \\ DIFFERENTIAL-ALGEBRAIC EQUATIONS AND ITS APPLICATIONS IN SOLVING TPPC PROBLEMS," Journal of Marine \\ Science and Technology. Vol. 19: Iss. 1, Article 9. \\ DOI: $10.51400 / 2709-6998.2139$ \\ Available at: https://jmstt.ntou.edu.tw/journal/vol19/iss1/9 \\ This Research Article is brought to you for free and open access by Journal of Marine Science and Technology. It has been \\ accepted for inclusion in Journal of Marine Science and Technology by an authorized editor of Journal of Marine Science and \\ Technology.
}




\title{
NUMERICAL SOLUTIONS OF DIFFERENTIAL-ALGEBRAIC EQUATIONS AND ITS APPLICATIONS IN SOLVING TPPC PROBLEMS
}

\author{
He-Sheng Wang*, Wei-Lun Jhu**, Chee-Fai Yung**, and Ping-Feng Wang***
}

Key words: differential-algebraic equations, computer algebra, trajectory-prescribed path control.

\begin{abstract}
In this paper, we present a numerical method for solving nonlinear differential algebraic equations (DAE's) based on the backward differential formulas (BDF) and the Pade series. Usefulness of the method is then illustrated by a numerical example, which is concerned with the derivation of the optimal guidance law for spacecraft. This kind of problems is called trajectory-prescribed path control (TPPC) in the literature. We reformulate the problem as a Hamiltonian DAE system (usually with a higher index). After establishing the system of spacecraft dynamics, we can derive the optimal guidance law of the system by the proposed numerical method.
\end{abstract}

\section{INTRODUCTION}

In system theory, a dynamical system is often considered as a set of ordinary differential or difference equations (ODE); these equations describe the relations between the system variables. As pointed out in [4], for the most general purpose of system analysis, one usually begins by defining the first order system

$$
\boldsymbol{F}(\dot{\boldsymbol{y}}(t), \boldsymbol{y}(t), t)=0,
$$

where $\boldsymbol{F}$ and $\boldsymbol{y}$ are vector-valued functions. Eq. (1) is termed as differential-algebraic equations (DAE), since it contains differential equations as well as a set of algebraic constraints. For control and systems engineers, it is usually assumed that

Paper submitted 02/03/09; revised 10/11/09; accepted 01/15/10. Author for correspondence: He-Sheng Wang (e-mail: hswang@mail.ntou.edu.tw)

*Department of Communications, Navigation and Control Engineering National Taiwan Ocean University, Keelung 202, Taiwan.

**Department of Electrical Engineering, National Taiwan Ocean University, Keelung, Taiwan.

***Institute for Information Industry, Taipei, Taiwan.
(1) can be rewritten in an explicit form

$$
\dot{\boldsymbol{y}}(t)=\boldsymbol{f}(\boldsymbol{y}(t), t) .
$$

An ODE of the form (2) is called a state-variable (statespace) description in the systems and control society. Since then, theorems and design techniques being developed are largely based on (2). In fact, the state-variable descriptions have been the predominant tool in systems and control theory. While the representation (2) will continue to be very important, there has been an increasing interest in working directly with (1).

If (1) can, in principle, be rewritten as (2) with the same state variables $\boldsymbol{y}$, then it will be referred to as a system of implicit ODE's. In this paper, we are especially interested in those problems for which this rewriting is impossible or less desirable. We consider the general nonlinear DAEs which are linear in the derivative

$$
\boldsymbol{A}(\boldsymbol{y}(t)) \dot{\boldsymbol{y}}(t)+\boldsymbol{f}(\boldsymbol{y}(t), t)=0 .
$$

Suppose that $\frac{\partial \boldsymbol{A}(\boldsymbol{y})}{\partial \boldsymbol{y}}$ has a constant rank. Then, in principle, locally the system (3) can be put in the semi-explicit form

$$
\begin{aligned}
\dot{x}(t) & =f_{1}(x(t), u(t), t), \\
0 & =f_{2}(x(t), u(t), t) .
\end{aligned}
$$

A large class of physical systems can be modeled by this kind of DAEs. The paper of Newcomb et al. [24] gives many practical examples, including circuit and system design, robotics, neural network, etc., and presents an excellent review on nonlinear DAEs. Many other applications of DAEs as well as numerical treatments can be found in [4]. An existence and uniqueness theory for nonlinear DAEs has been well developed in [26] by exploiting their underlying differential geometric structure. Recently, Venkatasubramanian et al. [28] have extensively studied the bifurcation phenomena of DAEs. 
They have also thoroughly investigated feasibility regions in differential-algebraic systems. The notion of feasibility regions provides a natural gateway to the stability theory of DAEs.

There are several reasons to consider systems of the form (4), rather than try to rewrite it as an ODE. Of great importance, we point out that, when physical problems are simulated, the model often takes the form of a DAE. DAEs can be used to depict a collection of relationships between variables of interest and some of their derivatives, which may be treated as an algebraic constraint between the state variables. These relationships may even be generated automatically by a modeling or simulation program. In particular, the variables thus introduced usually have a physical significance. Changing the model to (2) may produce less meaningful state variables. If the original DAE can be solved directly, then it becomes easier for scientists or engineers to explore the effect of modeling changes and parameter variations. These advantages enable researchers to focus their attention on the physical problem of interest. On the other hand, although the state-space models are very useful, but the state variables thus introduced often do not provide a physical meaning $[8,27]$. Besides, some physical phenomena, like impulse, hysteresis which are important in circuit theory, cannot be treated properly in the state-space models [18, 29]. Differential-algebraic equations representation provides a suitable way to handle such problems. It has been proven in the literature that DAE systems have higher capability in describing a physical system [17, 24, 29]. In fact, DAE system models appear more convenient and natural than state-space models in large scale systems, economics, networks, power, neural systems and elsewhere [17, $20,24]$.

In this paper, we investigate some of the control problems that are well suitable for the DAE system framework. In particular, we show that the optimal control problem can be reformulated as a Hamiltonian DAE. The derived DAE can then be solved by numerical methods. The numerical method is mainly based on the backward differential formulas (BDF) and the Pade series, which can be obtained by using computer algebra systems (such as MAPLE, Mathematica, etc.). Furthermore, we consider a practical design problem, namely the trajectory prescribed path control (TPPC) problem. In the simulation of space vehicles, we often encounter the TPPC problems. That is, we usually append a set of path constraints to the equations of motion for describing the shape of the trajectory. Here the optimal guidance law for aircraft dynamics is derived based on a DAE approach. After establishing the system of aircraft dynamics, we can derive the optimal guidance law of the system by solving a Hamiltonian DAE.

The rest of the paper is organized as follows: In Section 2, some of the elementary materials concerning DAE's are investigated. In Section 3, by using the Lagrange multiplier's method, it is shown that the optimal control problem can be rewritten as a Hamiltonian DAE. Simulation results are given in Section 4. Finally, some concluding remarks are given in Section 5.

\section{ELEMENTS OF DAES}

In this section we summarize some basic definitions and preliminary results that will be needed through-out this paper. Most of the treatments are purely algebraic and the definitions are fairly standard. First, the definitions of solvability and index for the general nonlinear DAE's (1) will be given in this section. Suppose that the DAE (1) consists of a system of $m$ equations in the $(2 m+1)$-dimensional variable $(\dot{\boldsymbol{y}}(t), \boldsymbol{y}(t), t)$. A function $\boldsymbol{y}(t)$ is said to be a solution of the DAE (1) on a interval $\mathcal{T}$ if $\boldsymbol{y}(t)$ is continuously differentiable on $\mathcal{T}$ and satisfies (1) for all $t \in \mathcal{T}$. The precise definition is given in the following.

Definition 1. Let $\mathcal{T}$ be an open subinterval of $\mathbb{R}, \Omega$ a connected open subinterval of $\mathbb{R}^{2 m+1}$, and $\mathbf{F}$ a differentiable function from $\Omega$ to $\mathbb{R}^{m}$. Then DAE (1) is solvable on $\mathcal{T}$ in $\Omega$ if there is an $r$-dimensional family of solutions $\phi(t, c)$ defined on a connected open set $\mathcal{T} \times \tilde{\Omega}, \tilde{\Omega} \subset \mathbb{R}^{r}$ such that:

1. $\phi(t, c)$ is defined on all of $\mathcal{T}$ for each $c \in \tilde{\Omega}$,

2. $(t, \phi(t, c), \dot{\phi}(t, c)) \in \Omega$ for $(t, c) \in \mathcal{T} \times \tilde{\Omega}$,

3. If $\psi(t)$ is any other solution with $(t, \psi(t, \boldsymbol{c}), \dot{\psi}(t, \boldsymbol{c})) \in \Omega$, then $\psi(t)=\phi(t, c)$ for some $c \in \tilde{\Omega}$,

4. The graph of $\phi$ as a function of $(t, c)$ is an $(r+1)$ - dimensional manifold.

The above definition means that locally there is an $r$-dimensional family of solutions. At any time $t_{0} \in \mathcal{T}$, the initial conditions form an $r$-dimensional manifold $\phi\left(t_{0}, c\right)$ and $r$ is independent of $t_{0}$. The solutions are a continuous function of the initial conditions on this manifold.

It is known that the property of index [4] plays a key role in the classification and behavior of DAE systems. It also provides a measurable scheme of the singularity of a DAE and the difficulty for numerically solving a DAE. The definition of index is described in the following.

Definition 2. The minimum number of times that all or part of DAE (1) must be differentiated with respect to $t$ in order to determine $\dot{\boldsymbol{y}}(t)$ as a continuous function of $\boldsymbol{y}(t)$ and $t$, is the index $v$ of the DAE (1).

The definition of the index given above is usually referred to as the differentiation index of DAE's. According to the definition, an implicit ODE has index zero. A DAE having index two or greater is referred to as a higher index DAE.

An alternative, but less general, characterization of the index is the number of iterations of the following (theoretical) procedure needed to convert the DAE into an ODE:

1. If $\partial \boldsymbol{F} / \partial \dot{\boldsymbol{y}}$ is nonsingular, then stop,

2. Suppose that $\partial \boldsymbol{F} / \partial \dot{\boldsymbol{y}}$ has constant rank and that nonlinear coordinate changes make (1) semi-explicit. Differentiate the constraint equation, let $\boldsymbol{F}=\mathbf{0}$ denote the new DAE, and return to 1 . 
To motivate the next definition, suppose that we wish to solve (1) by the implicit Euler method starting at time $t_{0}$ with constant step-size $h$. Let $t_{n}=t_{0}+n h, \boldsymbol{y}_{0}$ be the estimate for $\boldsymbol{y}\left(t_{n}\right)$. Then the implicit Euler method applied to (1) gives

$$
\boldsymbol{F}\left(t_{n}, \boldsymbol{y}_{n}, \frac{\boldsymbol{y}_{n}-\boldsymbol{y}_{n-1}}{h}\right)=0,
$$

which will need to be solved by a nonlinear equation solver. The Jacobian of $\boldsymbol{F}$ in (5) with respect to $\boldsymbol{y}_{n}$ is $\left(\frac{1}{h}\right) \boldsymbol{F}_{\boldsymbol{y}}+\boldsymbol{F}_{\boldsymbol{y}}$ so that this matrix pencil will be important. For the linear case, this was the pencil $\left(\frac{1}{h}\right) \boldsymbol{A}+\boldsymbol{B}$, where $\boldsymbol{A}, \boldsymbol{B}$ are constant matrices. Another definition of the index is given in the following in terms of the matrix pencil.

Definition 3. The local index $v_{1}$ of $(1)$ at $(\hat{t}, \hat{\boldsymbol{y}}, \dot{\hat{\boldsymbol{y}}})$ is the index of the matrix pencil $\lambda \boldsymbol{F}_{\dot{y}}(\hat{t}, \hat{\boldsymbol{y}}, \dot{\hat{\boldsymbol{y}}})+\boldsymbol{F}_{\boldsymbol{y}}(\hat{t}, \hat{\boldsymbol{y}}, \dot{\hat{\boldsymbol{y}}})$.

Proposition 4. Suppose that has constant rank. Then $v=1$ if and only if $v_{l} \equiv 1$.

From the above definition and proposition, it can be seen that the semi-explicit DAE

$$
\begin{gathered}
\dot{\boldsymbol{x}}_{1}=\boldsymbol{F}_{1}\left(\boldsymbol{x}_{1}, \boldsymbol{x}_{2}, t\right) \\
0=\boldsymbol{F}_{2}\left(\boldsymbol{x}_{1}, \boldsymbol{x}_{2}, t\right)
\end{gathered}
$$

has index one if and only if $\partial \boldsymbol{F}_{2} / \partial \boldsymbol{x}_{2}$ is nonsingular.

\section{NUMERICAL SOLUTIONS OF DAES}

In this section we examine some of the properties of the numerical methods for solving DAE's. We examine the linear multistep methods (LMM's) applied to DAE's, especially the most popular and hence best understood class of LMM's, namely the backward differentiation formulas (BDF). On the other hand, we also introduce a method that is well suitable for using computer algebra systems, namely the Pade approximation method. First we calculate power series of the given equations system (by using BDF) and then transform it into Pade series form, which give an arbitrary order algorithm for solving differential-algebraic equations numerically.

The first general technique for the numerical solution of DAE's, proposed in 1971 by Gear in a well-known and often cited paper [11], utilized the BDF. This method was initially defined for systems of differential equations coupled to algebraic equations

$$
\begin{gathered}
\dot{x}=\boldsymbol{f}(\boldsymbol{x}, \boldsymbol{u}, t), \\
0=\boldsymbol{g}(\boldsymbol{x}, \boldsymbol{u}, t),
\end{gathered}
$$

where $\boldsymbol{u}$ is a vector of the same dimension as $\boldsymbol{g}$. The algebraic variables $\boldsymbol{y}$ are treated in the same way as the differential variables $\boldsymbol{x}$ for BDF, and the method was soon extended to apply to any fully-implicit DAE system

$$
\boldsymbol{F}(\dot{\boldsymbol{y}}, \boldsymbol{y}, t)=0 .
$$

The simplest first order BDF method is the implicit Euler method, which consists of replacing the derivative in (8) by a backward difference

$$
\boldsymbol{F}\left(\frac{\boldsymbol{y}_{n}-\boldsymbol{y}_{n-1}}{h}, \boldsymbol{y}_{n}, t_{n}\right)=\mathbf{0},
$$

where $h=t_{n}-t_{n-1}$. The resulting system of nonlinear equations for $\boldsymbol{y}_{n}$ at each time step is then usually solved by Newton's method. The $k$-step (constant step-size) BDF consists of replacing $\dot{\boldsymbol{y}}$ by the derivative of the polynomial which interpolates the computed solution $k+1$ times $t_{n}, t_{n-1}, \ldots, t_{n-k}$, evaluated at $t_{n}$. This yields

$$
\boldsymbol{F}\left(\frac{\rho \boldsymbol{y}_{n}}{h}, \boldsymbol{y}_{n}, t_{n}\right)=\mathbf{0},
$$

where $\rho \boldsymbol{y}_{n}=\sum_{i=0}^{k} \alpha_{i} \boldsymbol{y}_{n-i}$ and $\alpha_{i}, i=0,1, \ldots, k$ are the coefficients of the BDF method. The $k$-step BDF method is stable for ODE's for $k<7$. The relation $\rho \boldsymbol{y}_{n}=\sum_{i=0}^{k} \alpha_{i} \boldsymbol{y}_{n-i}$ simply says that the derivative of $\boldsymbol{y}$ at $t_{n}$ can be represented as a polynomial of order $k$. We define another type of power series in the form

$$
f(t)=f_{0}+f_{1} t+f_{2} t^{2}+\cdots+\left(f_{n}+p_{1} e_{1}+\cdots+p_{m} e_{m}\right) t^{n},
$$

where $p_{1}, p_{2}, \ldots, p_{m}$ are constants, $e_{1}, e_{2}, \ldots, e_{m}$ are bases of vector $\boldsymbol{e}, m$ is the size of vector $\boldsymbol{e}$. Let $\boldsymbol{y}$ be a vector with $m$ elements, then every element can be represented by the Power series in (10). Therefore we can write

$$
y_{i}=y_{i, 0}+y_{i, 1} t+y_{i, 2} t^{2}+\cdots+e_{i} t^{n}
$$

where $y_{i}$ is the $i$ th element of $\boldsymbol{y}$. Substitute (11) into (1), we can get

$$
f_{i}=\left(f_{i, n}+p_{i, 1} e_{1}+\ldots+p_{i, m} e_{m}\right) t^{n-j}+Q\left(t^{n-j+1}\right)
$$

where $f_{i}$ is the $i$ th element of $\boldsymbol{F}(\dot{\boldsymbol{y}}, \boldsymbol{y}, t)$ in (1), and $Q\left(t^{n-j+1}\right)$ is a polynomial whose degree is no less than $t^{n-j+1}$. Now suppose that $\left(\dot{\boldsymbol{y}}_{0}, \boldsymbol{y}_{0}, t_{0}\right)$ is a set of consistent initial conditions for (1), i.e.

$$
\boldsymbol{F}\left(\dot{\boldsymbol{y}}_{0}, \boldsymbol{y}_{0}, t_{0}\right)=0
$$


Then the solutions of (1) can be assumed to be

$$
\boldsymbol{y}=\boldsymbol{y}_{0}+\boldsymbol{y}_{1} t+\boldsymbol{e} t^{2}
$$

where $\boldsymbol{e}$ is a vector function which is the same size as $\boldsymbol{y}_{0}$ and $\dot{\boldsymbol{y}}_{0}$. Substitute (13) into (1) and neglect higher order term, we have the linear equation of $\boldsymbol{e}$ in the form

$$
A e=B
$$

where $\boldsymbol{A}$ and $\boldsymbol{B}$ are constant matrices. Solving Eq. (14), the coefficient of $t^{2}$ in (13) can be determined. Repeating above procedure for higher order terms, we can get the arbitrary order power series of the solutions for (1).

From (11) and (14), we can determine the linear equation in (14) as follows:

$$
A_{i, j}=P_{i, j}
$$

Solve this linear equation, we have $e_{i}(i=1, \ldots, m)$. Substituting $e_{i}$ into (11), we then have $y_{i}(i=1, \ldots, m)$ which are polynomials of degree $n$. Repeating this procedure, we can get the arbitrary order Power series solution of differentialalgebraic equations in (1). The next step would be to put the power series into Pade series form and then obtain numerical solution of it.

Suppose that we are given a power series $\sum_{i=0}^{\infty} c_{i} x^{i}$, representing a function $f(x)$, so that

$$
f(x)=\sum_{i=0}^{\infty} c_{i} x^{i}
$$

where $c_{i}=0,1,2, \ldots$ is a given set of coefficients. This expansion is the fundamental starting point of any analysis using Pade approximants. A Pade approximant is a rational fraction

$$
[L / M]=\frac{a_{0}+a_{1} x+\cdots+a_{L} x^{L}}{b_{0}+b_{1} x+\cdots+b_{M} x^{M}}
$$

which has a MacLaurin expansion that is agreed with (16) as high (in order) as possible. For definiteness, we may take $b_{0}=$ 1. This choice turns out to be an essential part of the precise definition and (17) is our conventional notation with this choice for $b_{0}$. So there are $L+1$ independent numerator coefficients and $M$ independent denominator coefficients, making $L+M+1$ unknown coefficients in all. This number suggests that normally the $[L / M]$ ought to fit the power series (16) through the orders $1, x, x^{2}, \ldots, x^{L+M}$ in the notation of formal power series,

$$
\sum_{i=0}^{\infty} c_{i} x^{i}=\frac{a_{0}+a_{1} x+\cdots a_{L} x^{L}}{b_{0}+b_{1} x+\cdots+b_{M} x^{M}}+Q\left(x^{L+M+1}\right) .
$$

Upon cross-multiplying, the above equation can be re-arranged as

$$
\begin{aligned}
& \left(b_{0}+b_{1} x+\cdots+b_{M} x^{M}\right)\left(c_{0}+c_{1} x+\cdots\right) \\
& =a_{0}+a_{1} x+\cdots+a_{L} x^{L}+Q\left(x^{L+M+1}\right) .
\end{aligned}
$$

Equating the coefficients of $x^{L+1}, x^{L+2}, \ldots, x^{L+M}$ yields

$$
\begin{aligned}
b_{M} c_{L-M+1}+b_{M-1} c_{L-M+2}+\cdots+b_{0} c_{L+1} & =0, \\
b_{M} c_{L-M+2}+b_{M-1} c_{L-M+3}+\cdots+b_{0} c_{L+2} & =0, \\
& \vdots \\
b_{M} c_{L}+b_{M-1} c_{L+1}+\cdots+b_{0} c_{L+M} & =0 .
\end{aligned}
$$

If $j<0$, we define $c_{j}=0$ for consistency. Since $b_{0}=1$, Eq. (20) become a set of $M$ linear equations for $M$ unknown denominator coefficients:

$$
\left[\begin{array}{ccccc}
c_{L-M+1} & c_{L-M+2} & c_{L-M+3} & \cdots & c_{L} \\
c_{L-M+2} & c_{L-M+3} & c_{L-M+4} & \cdots & c_{L+1} \\
c_{L-M+3} & c_{L-M+4} & c_{L-M+5} & \cdots & c_{L+2} \\
\vdots & \vdots & \vdots & \vdots & \vdots \\
c_{L} & c_{L+1} & c_{L+2} & \cdots & c_{L+M+1}
\end{array}\right]\left[\begin{array}{c}
b_{M} \\
b_{M-1} \\
b_{M-2} \\
\vdots \\
b_{1}
\end{array}\right]=-\left[\begin{array}{c}
c_{L+1} \\
c_{L+2} \\
c_{L+3} \\
\vdots \\
c_{L+M}
\end{array}\right],
$$

from which the $b_{i}$ 's may be found. The numerator coefficients $a_{0}, a_{1}, \ldots, a_{L}$ follow immediately from (19) by equating the coefficients $1, x, x^{2}, \ldots, x^{L}$ :

$$
\begin{aligned}
& a_{0}=c_{0}, \\
& a_{1}=c_{1}+b_{1} c_{0}, \\
& a_{2}=c_{2}+b_{1} c_{1}+b_{2} c_{0}, \\
& \quad \vdots \\
& a_{L}=c_{L}+\sum_{i=1}^{\min (L, M)} b_{i} c_{L-i} .
\end{aligned}
$$

Thus, Eqs. (21) and (22) normally determine the Pade numerator and denominator and called the Pade equations. Summarizing the previous discussion, the thus constructed $[L / M]$ Pade approximant is able to agree with $\sum_{i=0}^{\infty} c_{i} x^{i}$ through order $x^{L+M}$.

Before the end of this section, we shall summarize the procedure for solving DAEs by using the proposed algorithm.

Algorithm Pade Series Solutions for DAEs

Input: The DAEs $\boldsymbol{F}(\dot{\boldsymbol{y}}(t), \boldsymbol{y}(t), t)=0$ and set an error tolerance $v$. 
Step 1: Calculate a power series solution for $\boldsymbol{y}(t)$ by using Eqs. (11)-(15). Denote the solution by $f(x)$.

Step 2: Calculate a Pade series solution for $f(x)$. Utilize Eq. (20)-(22) to compute the coefficients for the Pade series.

Step 3: Put the Pade series solution back into Eq. (1) to see if the error tolerance $v$ is fulfilled. If yes, then output $f(x)$; if not, then go back to Step $\mathbf{1}$ and calculate a solution with higher power.

End.

\section{HAMILTONIAN DAES}

In this section, we derive a class of DAE's by the Hamilton's principle. We shall call such a system the Hamiltonian DAE. The DAE structure allows a model to be obtained from energy functions, constraint equations, and a virtual work expression in a systematic manner. Hamilton's principle has its origin from the classical mechanics. It can, however, also arise naturally when dealing with the constrained optimization problem. Here we take the optimization viewpoint.

Consider a nonlinear time-varying dynamical system

$$
\dot{\boldsymbol{x}}(t)=\boldsymbol{f}(\boldsymbol{x}(t), \boldsymbol{u}(t), t)
$$

together with the associated performance index (cost function)

$$
J\left(t_{0}\right)=\int_{t_{0}}^{T} L(\boldsymbol{x}(t), \boldsymbol{u}(t), t) \mathrm{d} t,
$$

Where $\boldsymbol{x}(t) \in \mathbb{R}^{n}$ is the state, $\boldsymbol{u}(t) \in \mathbb{R}^{m}$ is the control input, $L(\boldsymbol{x}(t), \boldsymbol{u}(t), t)$ is the Lagrange function (or Lagrangian). The Lagrangian $L$ can be viewed as a weighting on the state variables and the control input in order to achieve a prescribed performance requirement. The main purpose of the control is to find an optimal feedback law $\boldsymbol{u}^{*}\left[t_{0}, T\right]$ such that the performance index (24) is minimized. Usually, in the classical mechanics, the Lagrangian is a function of the displacement variables $(\boldsymbol{x}, \boldsymbol{u})$ as well as the flows $(\dot{\boldsymbol{x}}, \dot{\boldsymbol{u}})$. However, in the control systems community, the Lagrangian is merely a function of the displacement. The Hamiltonian and the Lagrangian can be related by the Legendre transformation:

$$
H(\boldsymbol{x}(t), \boldsymbol{u}(t), t)=L(\boldsymbol{x}(t), \boldsymbol{u}(t), t)+\boldsymbol{p}^{T} \boldsymbol{f}(\boldsymbol{x}(t), \boldsymbol{u}(t), t),
$$

where $\boldsymbol{p}$ is the canonical momentum conjugated to $\boldsymbol{x}$. Using the above identity, Eq. (24) can be rewritten as:

$$
J^{\prime}=\int_{t_{0}}^{T}\left[H(\boldsymbol{x}(t), \boldsymbol{u}(t), t)-\boldsymbol{p}^{T}(t) \dot{\boldsymbol{x}}(t)\right] \mathrm{d} t .
$$

By using the Leibniz's rule, the increment in $J^{\prime}$ can be expressed in the increment of $\boldsymbol{x}, \boldsymbol{p}, \boldsymbol{u}$, and $t$ as follows:

$$
\mathrm{d} J^{\prime}=\int_{t_{0}}^{T}\left[H_{x}^{T} \delta \boldsymbol{u}-\boldsymbol{p}^{T} \delta \dot{\boldsymbol{x}}+\left(H_{\boldsymbol{p}}-\dot{\boldsymbol{x}}\right)^{T} \delta \boldsymbol{p}\right] \mathrm{d} t .
$$

To eliminate the variation in $\dot{\boldsymbol{x}}$, integrate by parts to get:

$$
-\int_{t_{0}}^{T} \boldsymbol{p}^{T} \delta \boldsymbol{x} \mathrm{d} t=-\left.\boldsymbol{p}^{T} \delta \boldsymbol{x}\right|_{T}+\left.\boldsymbol{p}^{T} \delta \boldsymbol{x}\right|_{t_{0}}+\int_{t_{0}}^{T} \dot{\boldsymbol{p}}^{T} \delta \boldsymbol{x} \mathrm{d} t
$$

Substituting (28) into (27) yields:

$$
\begin{aligned}
\mathrm{d} J^{\prime}= & -\left.\boldsymbol{p}^{T} \mathrm{~d} \boldsymbol{x}\right|_{T}+\left.\boldsymbol{p}^{T} \mathrm{~d} \boldsymbol{x}\right|_{t_{0}} \\
& +\int_{t_{0}}^{T}\left[\left(H_{x}+\dot{\boldsymbol{p}}\right)^{T} \delta \boldsymbol{x}+H_{u}^{T} \delta \boldsymbol{u}+\left(H_{\boldsymbol{p}}-\dot{\boldsymbol{x}}\right)^{T} \delta \boldsymbol{p}\right] \mathrm{d} t .
\end{aligned}
$$

According to the Lagrange theory, the constrained minimum of $J$ is attained at the unconstrained minimum of $J$. This is achieved when $\mathrm{d} J^{\prime}=0$ for all independent increments in its arguments. Setting to zero the coefficients of the independent increments $\delta \boldsymbol{x}, \delta \boldsymbol{u}$, and $\delta \boldsymbol{p}$ yields necessary conditions for a minimum $J$ as shown in the following.

$$
\begin{gathered}
\dot{\boldsymbol{x}}=\frac{\partial H}{\partial \boldsymbol{p}},-\dot{\boldsymbol{p}}=\frac{\partial H}{\partial \boldsymbol{x}}=\frac{\partial \boldsymbol{f}^{T}}{\partial \boldsymbol{x}} \boldsymbol{p}+\frac{\partial L}{\partial \boldsymbol{x}}, \\
0=\frac{\partial H}{\partial \boldsymbol{u}}=\frac{\partial L}{\partial \boldsymbol{u}}+\frac{\partial \boldsymbol{f}^{T}}{\partial \boldsymbol{u}} \boldsymbol{p} .
\end{gathered}
$$

The first two equations are the canonical Hamilton equations. The third equation can be viewed as an algebraic constraint. Therefore we shall call (30)-(31) a Hamiltonian DAE. The third equation is usually called the stationarity condition in the optimal control theory. The actual value of $\boldsymbol{p}(t)$ is by no means important, but it must evidently be determined as an intermediate step in finding the optimal control $\boldsymbol{u}^{*}(t)$, which depends on $\boldsymbol{p}(t)$ through the stationarity condition.

An important point is worth noting. The time derivative of the Hamiltonian is

$$
\begin{aligned}
\dot{H} & =\frac{\partial H}{\partial t}+\frac{\partial H^{T}}{\partial \boldsymbol{x}} \dot{\boldsymbol{x}}+\frac{\partial H^{T}}{\partial \boldsymbol{u}} \dot{\boldsymbol{u}}+\dot{\boldsymbol{p}}^{T} \boldsymbol{f} \\
& =\frac{\partial H}{\partial t}+\frac{\partial H^{T}}{\partial \boldsymbol{u}} \dot{\boldsymbol{u}}+\left(\frac{\partial H}{\partial \boldsymbol{x}}+\dot{\boldsymbol{p}}\right)^{T} \boldsymbol{f}
\end{aligned}
$$

If $\boldsymbol{u}(t)$ is an optimal control, then

$$
\dot{H}=\frac{\partial H}{\partial t} .
$$

Now, in the time-invariant case, $f$ and $L$ are not explicit function of $t$, and so neither is $H$. In this situation

$$
\dot{H}=0 \text {. }
$$


Hence, for time-invariant systems and cost functions, the Hamiltonian is a constant on the optimal trajectory.

\section{NUMERICAL EXAMPLE}

In this section, we first examine a classical trajectory prescribed path control (TPPC) problem which has been thoroughly studied in [3]. Then we will find an optimal guidance law for the TPPC problem by solving a Hamiltonian DAE. The two results are then compared in the end of the section.

The TPPC problems are often encountered in the simulation of space vehicle. In this problem, a vehicle is usually modeled to fly in space when its trajectory is imposed with constraints. That is, a set of path constraints are usually appended to the equations of motion for describing the shape of the trajectory. To model the system dynamics, we need a set of state (differential) equations

$$
\dot{\boldsymbol{x}}(t)=\boldsymbol{f}(\boldsymbol{x}(t), \boldsymbol{u}(t), t),
$$

that described the classical equations of motion together with a set of algebraic equations

$$
0=\boldsymbol{g}(\boldsymbol{x}(t), \boldsymbol{u}(t), t)
$$

that corresponds to the path constraints. In general, the $x$ variables are formed as state variables, while the $\boldsymbol{u}$ variables are treated as the control (algebraic) variables. The state variables describe the position, velocity, and possibly the mass of the vehicle. The control variables are typically described as angle of attack $(\alpha)$ and bank angle $(\beta)$, which effectively determine the magnitude and direction of the aerodynamic forces acting on the vehicle. In a TPPC problem the path constraints often are functions only of the state variables. In this paper, the TPPC problems of interest can be written as

$$
\begin{aligned}
\dot{\boldsymbol{x}}(t) & =\boldsymbol{f}(\boldsymbol{x}(t), \boldsymbol{u}(t), t), \\
0 & =\boldsymbol{g}(\boldsymbol{x}(t), t),
\end{aligned}
$$

where the matrix product $(\partial \boldsymbol{g} / \partial \boldsymbol{x})(\partial \boldsymbol{f} / \partial \boldsymbol{u})$ is nonsingular for all $t$ of interest. This condition is merely used to guarantee that the TPPC problem is solvable. See also [3].

The complete nonlinear kinematics of aircraft is considered in the TPPC problem. The state variables can be expressed in an earth centered relative coordinate system (see Fig. 1). In relative coordinate system, a spherical representation of the state variables can be utilized. Here we illustrate the representation of the state variables are altitude $h$, geocentric latitude $\lambda$, longitude $\psi$, magnitude of the relative velocity vector $V_{R}$, relative azimuth $A$, and relative fight path angle $\gamma$.

The control variables for TPPC problems are angle of attack and bank angle measured in a body coordinate system. The angle of attack is measured from the relative velocity

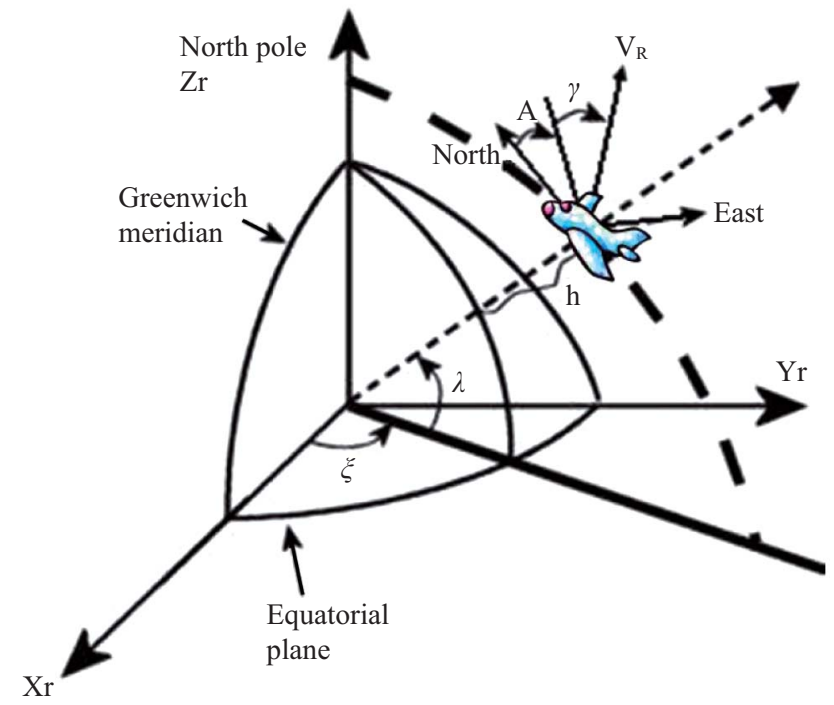

Fig. 1. State variables in relative coordinate.

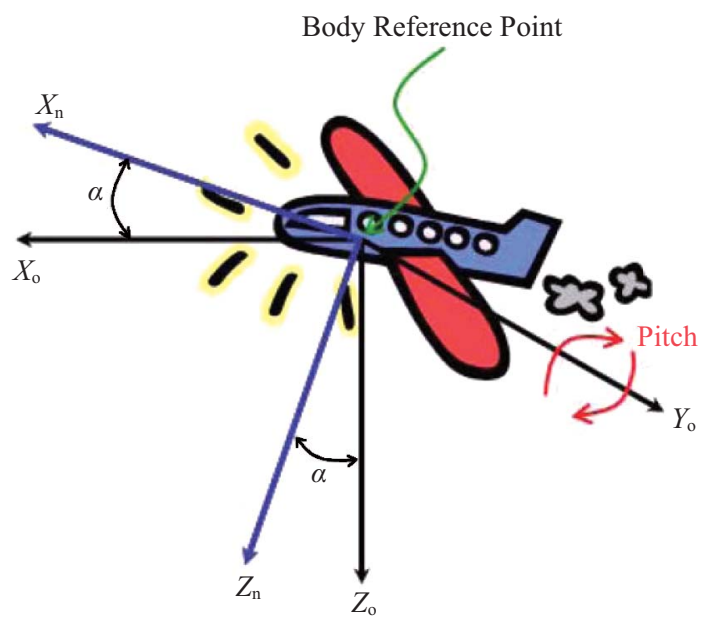

Fig. 2. Rotate about $Y_{O}$ through $\alpha$ in body coordinate system.

vector of the vehicle to the body $X_{O}$ axis, and corresponds to 'pitch' about the vehicle's latitudinal axis (namely the $Y_{O}$ axis) as shown in Fig. 2.

The bank angle is measured from the relative velocity $y$-axis to the body $Y_{O}$ axis, and corresponds to a 'roll' about the vehicle's longitudinal axis (namely the $X_{O}$ axis) as shown in Fig. 3.

For most trajectory application problems, position and velocity of the vehicle (namely the rectangular three-component vectors $\vec{r}$ and $\vec{v}$ ) are often expressed as the differential variables. The equations of aircraft dynamical system can be written as

$$
\begin{aligned}
\dot{\vec{r}} & =\vec{v}, \\
\dot{\vec{v}} & =\vec{g}(\vec{r})+\frac{1}{m}\left(\vec{F}_{A}(\vec{r}, \vec{v}, \vec{\alpha}, \vec{\beta})+\vec{F}_{P}(\vec{r}, \vec{\alpha}, \vec{\beta})\right),
\end{aligned}
$$




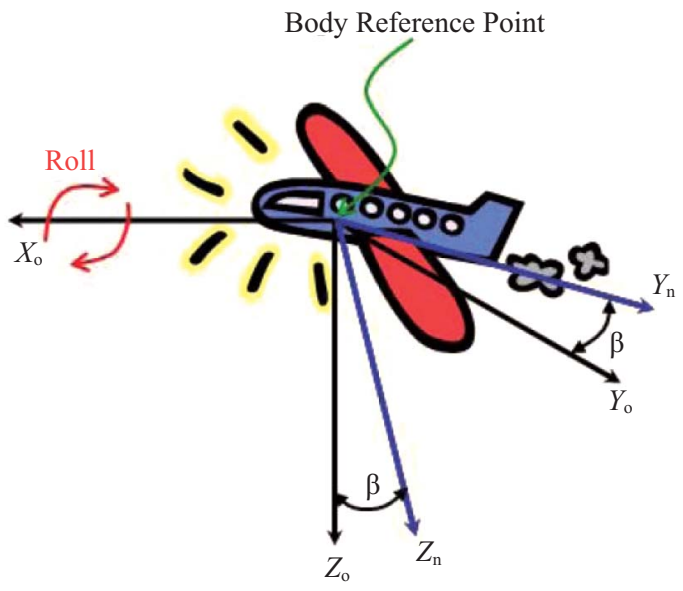

Fig. 3. Rotate about $X_{O}$ through $\beta$ in Body Coordinate System.

with $\vec{r}\left(t_{0}\right), \vec{v}\left(t_{0}\right)$ and $t_{0}$ given, and

$$
\begin{aligned}
\vec{g}(\vec{r}) & =\text { acceleration vector due to gravity } \\
m & =\text { mass of the vehicle, } \\
\vec{F}_{A}(\vec{r}, \vec{v}, \vec{\alpha}, \vec{\beta}) & =\text { aerodynamic force vector, } \\
\vec{F}_{P}(\vec{r}, \vec{\alpha}, \vec{\beta}) & =\text { propulsive force vector. }
\end{aligned}
$$

In this paper, we limit our discussion to reentry vehicles with no propulsive forces so that $\vec{F}_{P}(\vec{r}, \vec{\alpha}, \vec{\beta}) \equiv 0$, and simplify the simulation to model only a spherical geopotential and spherical earth. When state variables are expressed in relative coordinates, the differential equations of above can be translated to the following six equations of motion:

$$
\begin{aligned}
\dot{h}= & V_{R} \times \sin (\gamma), \\
\dot{\xi}= & \frac{V_{R} \times \cos (\gamma) \times \sin (A)}{r \times \cos (\lambda)}, \\
\dot{\lambda}= & \frac{V_{R}}{r} \times \cos (\gamma) \times \cos (A), \\
\dot{V}_{R}= & -\frac{D}{m}-g \times \sin (\gamma)-\Omega_{E}^{2} \times r \times \cos (\lambda) \\
& \times(\sin (\lambda) \cos (A) \cos (\gamma)-\cos (\lambda) \sin (\gamma)), \\
\dot{\gamma}= & \frac{L \cos (\beta)}{m \times V_{R}}+\frac{\cos (\gamma)}{V_{R}}\left(\frac{V_{R}^{2}}{r}-g\right) \\
& +2 \Omega_{E} \cos (\lambda) \sin (A) \\
& +\frac{r \Omega_{E}^{2} \cos (\lambda)}{V_{R}} \times(\sin (\lambda) \cos (A) \sin (\gamma) \\
& +\cos (\lambda) \cos (\gamma)),
\end{aligned}
$$

$$
\begin{gathered}
\dot{A}=\frac{L \sin (\beta)}{m V_{R}} \cos (\gamma)+\frac{V_{R}}{r} \cos (\gamma) \sin (A) \tan (\lambda) \\
-2 \Omega_{E}(\cos (\lambda) \cos (A) \tan (\gamma)-\sin (\lambda)) \\
+\frac{r \Omega_{E}^{2} \cos (\lambda) \sin (\lambda) \sin (A)}{V_{R} \cos (\gamma)}
\end{gathered}
$$

where

$$
a_{e}=\text { the earth radius, }
$$$$
r=H+a_{e},
$$$$
\mu=\text { gravitational constant, }
$$$$
g=\mu / r^{2} \text {, the gravity force, }
$$

$\Omega_{E}=$ earth angular rotation rate,

$m=$ the mass of the vehicle,

$S=$ the vehicle cross-sectional reference area, $\rho(h)=$ the atmospheric density,

$C_{L}(\alpha)=$ the aerodynamic lift coefficient,

$C_{D}(\alpha)=$ the aerodynamic drag coefficient,

$$
\begin{aligned}
& L=\frac{1}{2} \rho C_{L} S V_{R}^{2}, \text { the aerodynamic lift force, } \\
& D=\frac{1}{2} \rho C_{D} S V_{R}^{2}, \text { the aerodynamic drag force. }
\end{aligned}
$$

It's worth noting that the equations of motion are undefined if $\gamma=90^{\circ}$ or $\lambda=90^{\circ}$. The equation-prescribed path is usually written as functions of the relative state variables. When the state (differential) and algebraic equations are both expressed in relative coordinates, it will be easy to determine the index of the resulting DAE system. The index of the resulting DAE system in relative coordinates can be found by a reduction technique based on repeated differentiations of the algebraic equations. This procedure can reduce the index of the system to one. Here, the vehicle is solved by the 5-step backward differential formulas (BDF). First, let us consider a simple path constraint so that the vehicle is constrained to fly along a prescribed azimuth and fight path angle trajectory. It is given by the state variable constraints as follows:

$$
\begin{aligned}
& 0=\gamma+1+9 \times(t / 300)^{2}, \\
& 0=A-45-90 \times(t / 300)^{2} .
\end{aligned}
$$

The above path constraints are also discussed in [4]. The DAE system (36)-(37) has index two. We have to differentiate the algebraic Eq. (37) once and substitute for $A$ ' and $\gamma^{\prime}$ from the differential Eq. (36). Then the resulting index one DAE system related to the original system can be derived in the following, 


$$
\begin{array}{cc}
\dot{h}=V_{R} \times \sin (\gamma), & \text { system (38). Numerical results are obtai } \\
\text { set of initial values: } & \\
\dot{\xi}=\frac{V_{R} \cos (\gamma) \sin (A)}{r \cos (\lambda)}, & h=150000 \mathrm{ft} ., \\
\dot{\lambda}=\frac{V_{R}}{r} \cos (\gamma) \cos (A), & \xi=0^{\circ}, \\
V_{R}=-\frac{D}{m}-g \sin (\gamma) & \lambda=0^{\circ}, \\
V_{R} & =12000 \mathrm{ft} . / \mathrm{sec}, \\
-r \Omega_{E}^{2} \cos (\lambda) \times & \gamma=-1^{\circ}, \\
(\sin (\lambda) \cos (A) \cos (\gamma)-\cos (\lambda) \sin (\gamma)), & A=45^{\circ}, \\
& \alpha=2.6729^{\circ}, \\
& \beta=-0.0522^{\circ} .
\end{array}
$$$$
\dot{\gamma}=\frac{L \cos (\beta)}{m V_{R}}+\frac{\cos (\gamma)}{V_{R}}\left(\frac{V_{R}^{2}}{r}-g\right)
$$$$
+2 \Omega_{E} \cos (\lambda) \sin (A)
$$$$
+\frac{r \Omega_{E}^{2} \cos (\lambda)}{V_{R}} \times
$$$$
(\sin (\lambda) \cos (A) \sin (\gamma)+\cos (\lambda) \cos (\gamma)),
$$$$
\dot{A}=\frac{L \sin (\beta)}{m V_{R} \cos (\gamma)}+\frac{V_{R}}{r} \cos (\gamma) \sin (A) \tan (\lambda)
$$$$
-2 \Omega_{E}(\cos (\gamma) \cos (A) \tan (\lambda)-\sin (\lambda))
$$$$
+\frac{r \Omega_{E}^{2} \cos (\lambda) \sin (\lambda) \sin (A)}{V_{R} \cos (\gamma)},
$$$$
0=\frac{t}{5000}+\frac{L \cos (\beta)}{m V_{R}}+\frac{\cos (\gamma)}{V_{R}}\left(\frac{V_{R}^{2}}{r}-g\right)
$$$$
+2 \Omega_{E} \cos (\lambda) \sin (A)
$$$$
+\frac{r \Omega_{E}^{2} \cos (\lambda)}{V_{R}} \times
$$$$
(\sin (\lambda) \cos (A) \sin (\gamma)+\cos (\lambda) \cos (\gamma)),
$$$$
0=-\frac{t}{500}+\frac{L \sin (\beta)}{m V_{R} \cos (\gamma)}
$$$$
+\frac{V_{R}}{r} \cos (\gamma) \sin (A) \tan (\lambda)
$$$$
-2 \Omega_{E}(\cos (\lambda) \cos (A) \tan (\gamma)-\sin (\lambda))
$$$$
+\frac{r \Omega_{E}^{2} \cos (\lambda) \sin (\lambda) \sin (A)}{V_{R} \cos (\gamma)} .
$$

Consistent initial values for the DAE system are determined by selecting the initial differential and control variables to satisfy the two algebraic equations in the related index one

system (38). Numerical results are obtained for the following

For simplicity, we consider the problem in a model of spherical gravitational earth. So we can use the relative state variables in the differential equations. The related coefficients of the vehicle in this experiment are given as follows:

$$
\begin{aligned}
& m=2.8905 \text { tons, } \\
& S=550 \mathrm{ft}^{2}{ }^{2}
\end{aligned}
$$

The atmosphere is modeled with simple relation, $\rho=$ $0.002378 \exp (-h / 23800)$. The lift and drag coefficients are chosen as functions of angles of attack,

$$
\begin{aligned}
& C_{L}=(0.01) \alpha, \\
& C_{D}=0.04+0.1 C_{L}^{2} .
\end{aligned}
$$

The rest of the constraints needed in (36) are shown as follows:

$$
\begin{aligned}
a_{e} & =20902900 \mathrm{ft} ., \\
\mu & =1.4077 \times 10^{16} \mathrm{ft}^{3} / \mathrm{s}^{2}, \\
\Omega_{E} & =7.2921 \times 10^{-5} \mathrm{rad} / \mathrm{s} .
\end{aligned}
$$

In our simulation, a truth model is generated by solving the index one problem with a tight local relative tolerance (EPS = $10^{-8}$ ) and absolute tolerance (EPS $=10^{-7}$ ). The state variables of vehicle are plotted as functions of time in Fig. 4 and Fig. 5. Now we shall impose an optimal criterion on the TPPC problem so that the problem can be reformulated as a Hamiltonian DAE. Consider the following performance index:

$$
J\left(t_{0}\right)=\int_{t_{0}}^{T} L(\boldsymbol{x}(t), \boldsymbol{u}(t), t) \mathrm{d} t .
$$

The Lagrange function $L$ considered here has the following quadratic form:

$$
L=\frac{1}{2}\left(\boldsymbol{x}^{T} \boldsymbol{Q} \boldsymbol{x}+\boldsymbol{u}^{T} \boldsymbol{R} \boldsymbol{u}\right)
$$



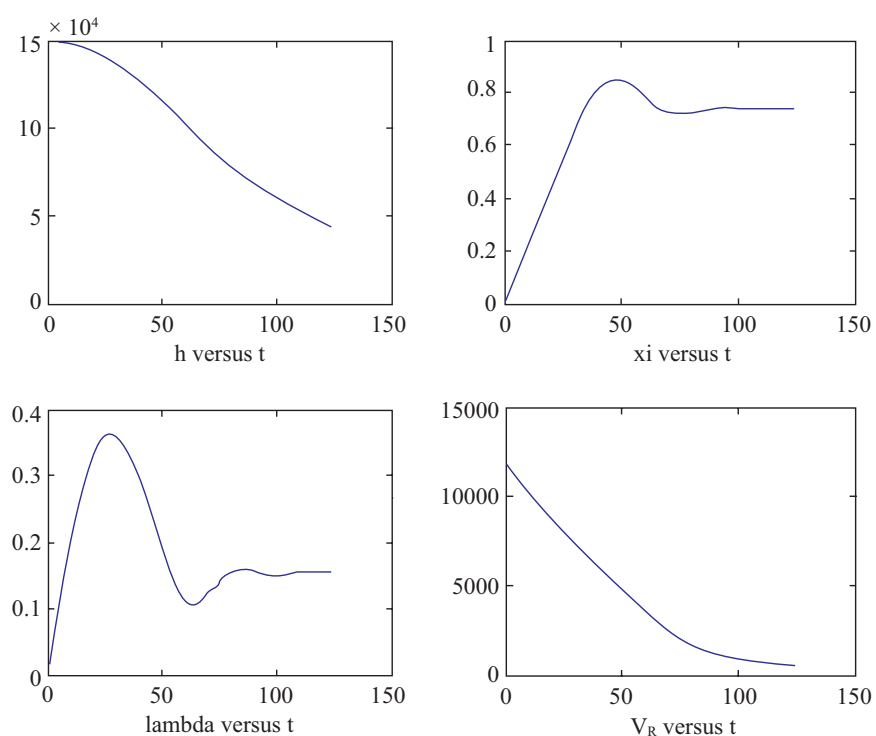

Fig. 4. State variables.

where the matrix $\boldsymbol{Q}$ is a $6 \times 6$ identity matrix and $\boldsymbol{R}$ is a $2 \times 2$ identity matrix, $\boldsymbol{x}$ is the state variable, and $\boldsymbol{u}$ is the control variable. To solve the optimal control problem is then equivalent to solving the following Hamiltonian DAE:

$$
\begin{gathered}
\dot{\boldsymbol{x}}=\frac{\partial H}{\partial \boldsymbol{p}}, \\
-\dot{\boldsymbol{p}}=\frac{\partial H}{\partial \boldsymbol{x}}, \\
0=\frac{\partial H}{\partial \boldsymbol{u}} .
\end{gathered}
$$

where $H$ is the Hamiltonian function for the aircraft dynamics, and $\boldsymbol{p}$ is the generalized momentum vector. As mentioned in Section 3, the Hamiltonian function $H$ is equal to $L+\boldsymbol{p}^{T} \boldsymbol{f}$. Here $f$ is the equations of the vehicular motion. In our experiment, $\dot{\boldsymbol{x}}=\partial H / \partial \boldsymbol{p}$ of the Hamiltonian DAE system is equal to the differential equations of the TPPC problem, namely the equations of motion in relative coordinates. Here we define six variables as generalized momentum to yield an appropriate stationary point with choosing state variables $\boldsymbol{x}$ and control variables $\boldsymbol{u}$. The control variables $\boldsymbol{u}$ are also the angle of attack $\alpha$ and bank angle $\beta$. Then $\dot{\boldsymbol{p}}=\partial H / \partial \boldsymbol{x}$ of the Hamiltonian DAE system is given as follows:

$$
\begin{aligned}
\dot{p}_{1}= & \frac{V_{R} \cos (\lambda) \sin (A) p_{2}}{\cos (\lambda)(h+20902900)^{2}}+\frac{V_{R} \cos (\gamma) \cos (A) p_{3}}{(h+20902900)^{2}} \\
& -1.4536 \times 10^{-5} \times\left(1.189 \times 10^{-8} \alpha^{2}+4.756 \times 10^{-5}\right) \\
& \times V_{R}^{2} p_{4} e^{-\frac{h}{23800}}
\end{aligned}
$$
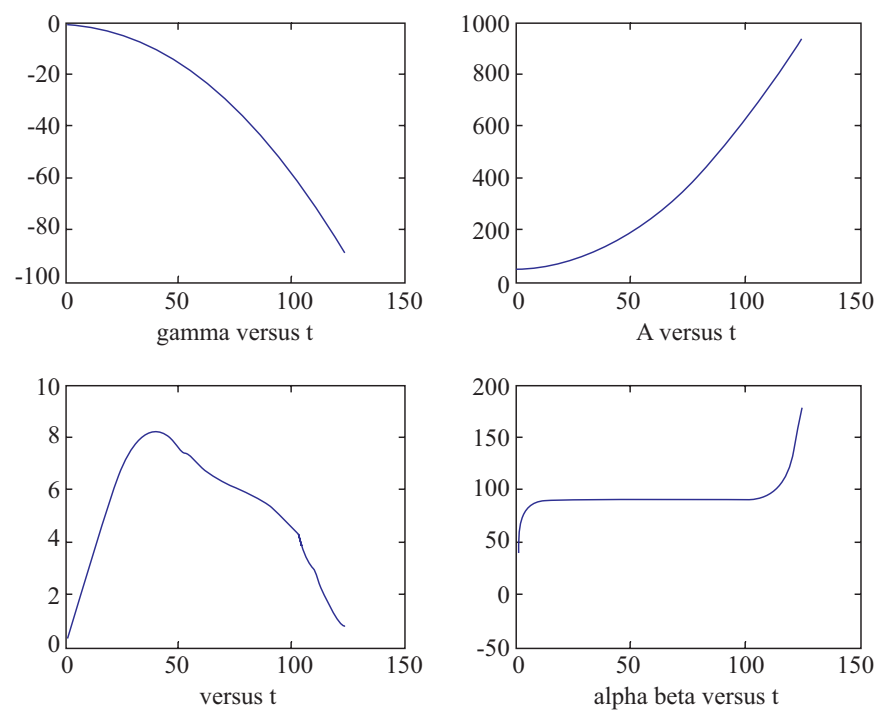

Fig. 5. State variables and control variables.

$$
\begin{aligned}
& -\frac{2.8153 \times 10^{16} p_{4}}{\sin (\gamma)(h+20902900)^{3}} \\
& +5.3175 \times 10^{-9} \cos (\lambda) p_{4} \\
& \times(\sin (\lambda) \cos (A) \cos (\gamma)-\cos (\lambda) \sin (\gamma)) \\
& +1.7283 \times 10^{-10} \alpha V_{R} \cos (\beta) p_{5} e^{-\frac{h}{23800}} \\
& -\cos (\gamma) p_{5}\left(-\frac{V_{R}^{2}}{(h+20902900)^{2}}+\frac{2.8153 \times 10^{16}}{V_{R}(h+20902900)^{3}}\right) \\
& +5.3175 \times 10^{-9} \frac{\cos (\lambda) p_{5}}{V_{R}}
\end{aligned}
$$

$\times(\sin (\lambda) \cos (A) \sin (\gamma)+\cos (\lambda) \cos (\gamma))$

$$
+1.7283 \times 10^{-10} \frac{\alpha V_{R} \sin (\beta)}{\cos (\gamma) p_{6}} e^{-\frac{h}{23800}}
$$$$
+\frac{V_{R} \cos (\gamma) \sin (A) \tan (\lambda) p_{6}}{(h+20902900)^{2}}
$$$$
+5.3175 \times 10^{-9} \frac{\cos (\lambda) \sin (\lambda) \sin (A) p_{6}}{V_{R} \cos (\gamma)}-h,
$$

$\dot{p}_{2}=-\xi$

$\dot{p}_{3}=-\frac{V_{R} \cos (\gamma) \sin (A) \sin (\lambda) p_{2}}{\cos ^{2}(\lambda)(h+20902900)}$

$-\left(5.3175 \times 10^{-9} h+1.1115\right) \sin (\lambda) p_{4}$

$\times(\sin (\lambda) \cos (A) \cos (\gamma)-\cos (\lambda) \sin (\gamma))$ 

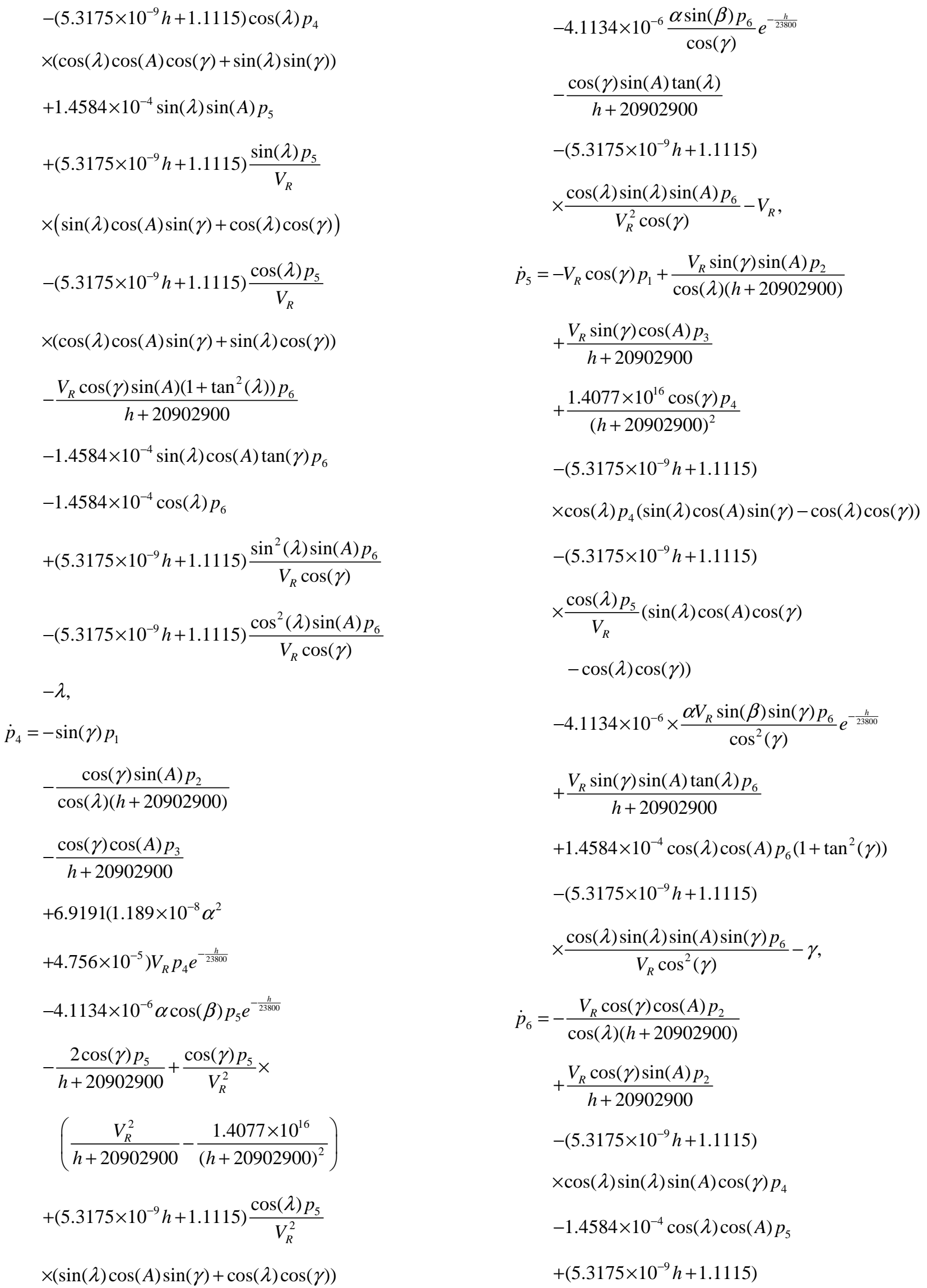

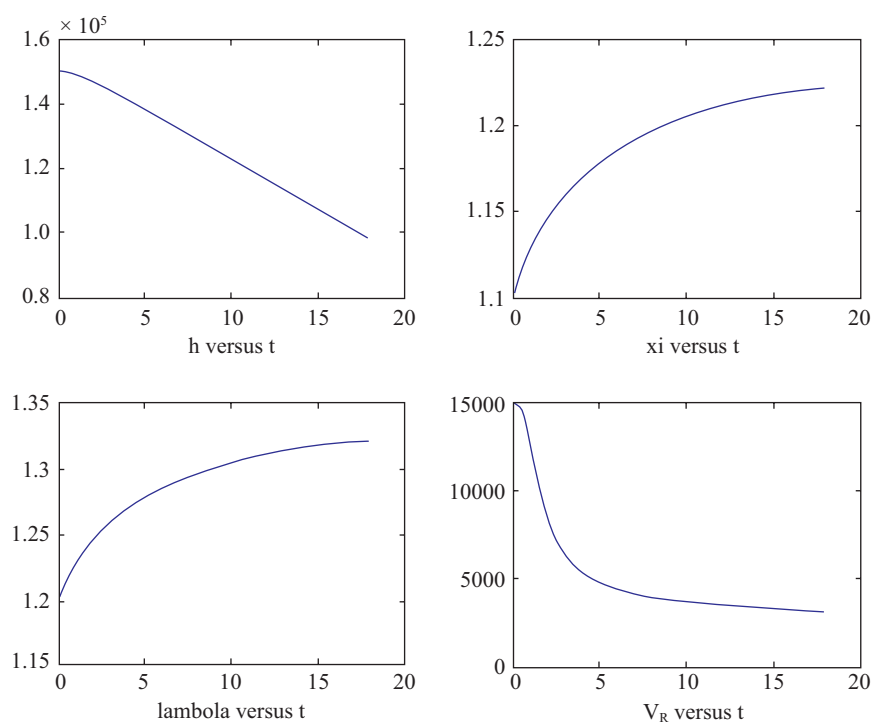

Fig. 6. State variables.

$$
\begin{aligned}
& \times \frac{\cos (\lambda) \sin (\lambda) \sin (A) \sin (\gamma) p_{5}}{V_{R}} \\
& -\frac{V_{R} \cos (\gamma) \cos (A) \tan (\lambda) p_{6}}{h+20902900} \\
& -1.4584 \times 10^{-4} \cos (\lambda) \sin (A) \tan (\gamma) p_{6} \\
& -\left(5.3175 \times 10^{-9} h+1.1115\right) \\
& \times \frac{\cos (\lambda) \sin (\lambda) \cos (A) p_{6}}{V_{R} \cos (\gamma)}-A .
\end{aligned}
$$

The stationarity conditions are shown below:

$$
\begin{aligned}
0= & -8.2269 \times 10^{-9} \alpha V_{R}^{2} p_{4} e^{-\frac{h}{23800}} \\
& +4.1134 \times 10^{-6} V_{R} \cos (\beta) p_{5} e^{-\frac{h}{23800}} \\
& +4.1134 \times 10^{-6} \frac{V_{R} \sin (\beta) p_{6}}{\cos (\gamma)} e^{-\frac{h}{23800}} \\
& +\alpha, \\
0= & -4.1134 \times 10^{-6} \alpha V_{R} \sin (\beta) p_{5} e^{-\frac{h}{23800}} \\
& +4.1134 \times 10^{-6} \frac{V_{R} \cos (\beta) p_{6}}{\cos (\gamma)} e^{-\frac{h}{23800}} \\
& +\beta .
\end{aligned}
$$

The three parts of the Hamiltonian DAEs (36) (41) (42) can be put into the following compact form:
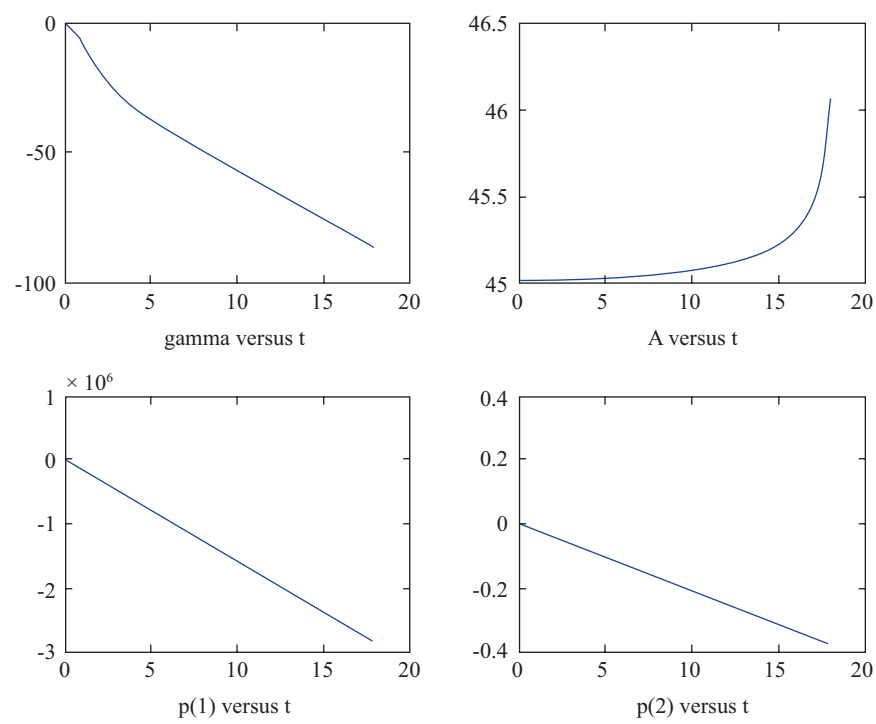

Fig. 7. State variables and generalized momentum.

$$
\left[\begin{array}{lll}
\boldsymbol{I} & 0 & 0 \\
0 & \boldsymbol{I} & 0 \\
0 & 0 & 0
\end{array}\right] \dot{\boldsymbol{y}}=\left[\begin{array}{c}
\frac{\partial H}{\partial \boldsymbol{p}} \\
\frac{\partial H}{\partial \boldsymbol{x}} \\
\frac{\partial H}{\partial \boldsymbol{u}}
\end{array}\right]
$$

where $\boldsymbol{y}$ is equal to $\left[\begin{array}{l}\boldsymbol{x} \\ \boldsymbol{p} \\ \boldsymbol{u}\end{array}\right]$. Numerical results are obtained for the following set of initial values:

$$
\begin{array}{ll}
h=150070 \mathrm{ft} ., & \xi=1.1^{\circ}, \\
\lambda=1.2^{\circ}, & V_{R}=15000 \mathrm{ft} . / \mathrm{sec}, \\
\gamma=-1^{\circ}, & A=45^{\circ}, \\
p_{1}=2000, & p_{2}=10^{-6}, \\
p_{3}=-10, & p_{4}=200, \\
p_{5}=2000, & p_{6}=8.9, \\
\alpha=2.6725^{\circ}, & \beta=-0.0522^{\circ} .
\end{array}
$$

The numerical results are shown in Figs. 6-9.

For the above two cases, we make the following observation. According to the above numerical results, we can see that the control variable $\alpha$ in the optimal control problem decreases along with time in compared with the simple TPPC problem. Although $\beta$ increase slightly, it still doesn't affect the value of Lagrange function $L$, so as to attain the minimum. From those figures, it can be seen that the values of $h, V_{R}$, $\gamma$ decrease severely and the values of $\lambda, \xi$, and $A$ increase slowly 

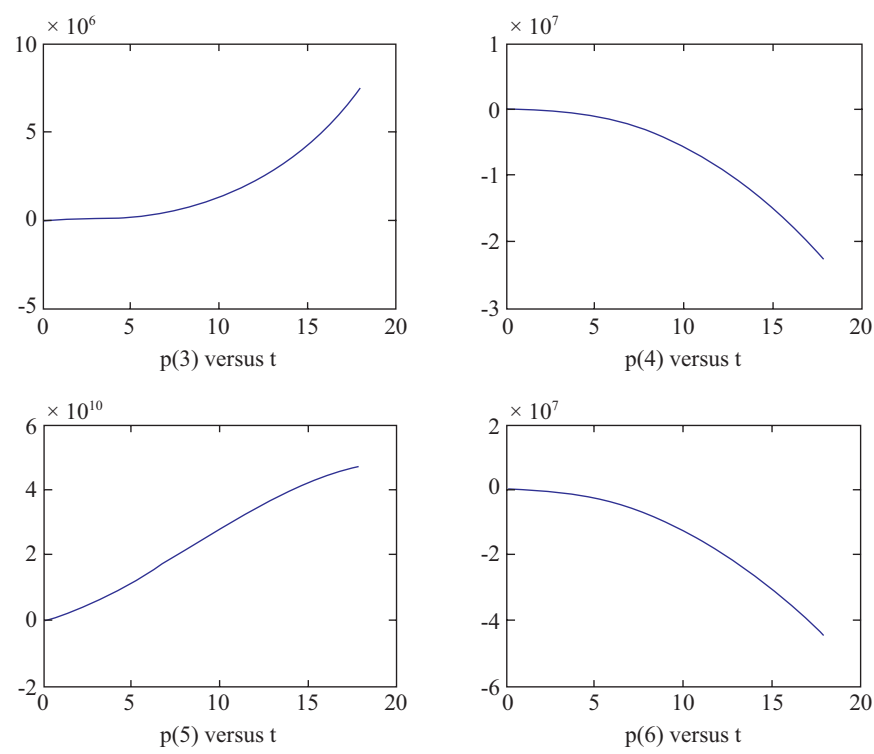

Fig. 8. Generalized momentum.
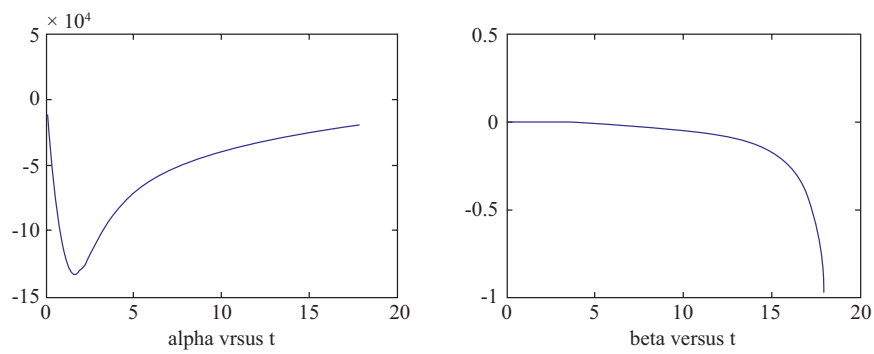

Fig. 9. Generalized momentum and control variables.

in order to meet the performance requirement, namely the state variables must have the minimum energies among all possible trajectories. For the two cases, it is calculated, in the same time interval, that the Lagrangian $L$ is equal to $2.906314888887725 \times 10^{15}$ for the optimal control problem, and equal to $3.950072046453987 \times 10^{15}$ for the TPPC problem. In other words, the TPPC problem with optimal control design can not only achieve the purpose of flight control, but also satisfy the minimum energy requirement.

\section{CONCLUSIONS}

We have proposed a potential unified framework for solving optimal control problem in the present paper. The optimal control problem is reformulated as a Hamiltonian DAE and then is solved by the BDF method. To serve as an illustrative example, we also derive an optimal control law for the TPPC problem. Currently, we just limited the fight path angle $\gamma$ and the azimuth $A$ so that $\gamma$ was gradually decreasing from $-1^{\circ}$ and $A$ increasing from $45^{\circ}$. It is possible to alter the algebraic constraint to a more complex equation or a realistic fight route.

\section{REFERENCES}

1. Ailon, A., "Controllability of generalized linear time-invariant systems," IEEE Transactions on Automatic Control, Vol. AC-32, No. 5, pp. 429-432 (1987).

2. Ascher, U. M. and Petzold, L. R., Computer Methods for Ordinary Differential Equations and Differential-Algebraic Equations, Society for Industrial and Applied Mathematics, Philadelphia (1998).

3. Brenan, K. E., Stability and Convergence of Difference Approximations for Higher Index Differential-Algebraic Systems with Applications in Trajectory Control, Ph.D. Thesis, Department of Mathematics, University of California, Los Angeles (1983).

4. Brenan, K. E., Campbell, S. L., and Petzold, L. R., Numerical Solution of Initial-Value Problems in Differential-Algebraic Equations, SIAM's Classics in Applied Mathematics Series, Society for Industrial and Applied Mathematics, Philadelphia (1996).

5. Campbell, S. L., Singular Systems of Differential Equations I, Pitman, New York (1980).

6. Campbell, S. L., Singular Systems of Differential Equations II, Pitman, New York (1982).

7. Dziurla, B. and Newcomb, R. W., "The Drazin inverse and semi-state equations," Proceedings of the International Symposium on Mathematical Theory of Networks and Systems, Delft, pp. 283-289 (1979).

8. Fang, C. H. and Chang, F. R., "Analysis of stability robustness for generalized state-space systems with structured perturbations," Systems and Control Letters, Vol. 21, No. 2, pp. 109-114 (1993).

9. Lewis, F. L. and Syrmos, V. L., Optimal Control, John Wiley and Sons, Inc., New York (1995).

10. Gantmacher, F. R., The Theory of Matrices, Vol. I and II, Chelsea, New York (1959).

11. Gear, C. W., "The simultaneous numerical solution of differentialalgebraic equations," IEEE Transactions on Circuit Theory, Vol. CT-18, No. 1, pp. 89-95 (1971).

12. Gear, C. W., "Differential-algebraic equation index transformations," SIAM Journal on Scientific and Statistical Computing, Vol. 9, pp. 39-47 (1988).

13. Hayton, G. E., Fretwell, P., and Pugh, A. C., "Fundamental equivalence of generalized state space systems," IEEE Transactions on Automatic Control, Vol. AC-31, No. 5, pp. $431-439$ (1986).

14. Karcanias, N., "Regular state-space realizations of singular system control problems," Proceedings of the 28th Conference on Decision and Control, Los Angeles, CA, pp. 1144-1146 (1987).

15. Klamm, Jr., C. F., Anderson, B. D. O., and Newcomb, R. W., "Stability of passive time-variable circuits," Proceedings of the Institution of Electrical Engineers, Vol. 114, No. 1, pp. 71-75 (1967).

16. Kucera, V., "Stationary LQG control of singular systems," IEEE Transactions on Automatic Control, Vol. AC-31, No. 1, pp. 31-39 (1986).

17. Lewis, F. L., "A survey of linear singular systems," Circuits, Systems, and Signal Process, Vol. 5, No. 1, pp. 3-36 (1986).

18. Lewis, F. L., "A tutorial on the geometric analysis of linear time-invariant implicit systems," Automatica, Vol. 28, No. 1, pp. 119-137 (1992).

19. Lotstedt, P. and Petzold, L., "Numerical solution of nonlinear differential equations with algebraic constraints, I: convergence results for backward differentiation formulas," Mathematics of Computation, Vol. 46, No. 174, pp. 491-516 (1986).

20. Luenberger, D. G., "Dynamic equations in descriptor form," IEEE Transactions on Automatic Control, Vol. AC-22, No. 3, pp. 312-321 (1977).

21. Luenberger, D. G., "Non-linear descriptor systems," Journal of Economic Dynamics and Control, Vol. 1, pp. 212-242 (1979).

22. Marz, R., "On initial value problems in differential-algebraic equations and their numerical treatment," Computing, Vol. 35, No. 1, pp. 13-37 (1985).

23. McClamroch, N. H., "Singular systems of differential equations as dynamic models for constrained robot systems," Proceedings of the IEEE Conference on Robotics and Automations, San Francisco, California, pp. 21-28 (1986). 
24. Newcomb, R. W. and Dziurla, B., "Some circuits and systems applications of semistate theory," Circuits, Systems and Signal Processing, Vol. 8, No. 3, pp. 235-260 (1989).

25. Petzold, L., "Numerical solution of differential/algebraic systems by implicit Runge-Kutta methods," Proceedings of the 27th Midwest Symposium on Circuits and Systems, Morgantown, West Virginia, pp. 678691 (1984).

26. Reich, S., "On an existence and uniqueness theory for nonlinear differential-algebraic equations," Circuits, Systems and Signal Processing, Vol. 10, No. 3, pp. 343-359 (1991).

27. Takaba, K., Morihira, N., and Katayama, T., " $H_{\infty}$ control for descriptor systems - a $J$-spectral factorization approach," Proceedings of 33rd Conference on Decision and Control, Lake Buena Vista, Florida, pp. 22512256 (1994).

28. Venkatasubramanian, V., Schattler, H., and Zaborszky, J., "Local bifurcations and feasibility regions in differential-algebraic systems," IEEE Transactions on Automatic Control, Vol. AC-40, No. 12, pp. 1992-2013 (1995).

29. Verghese, G., Levy, B. C., and Kailath, T., "A generalized state-space for singular systems," IEEE Transaction on Automatic Control, Vol. AC-26, No. 4, pp. 811-831 (1981). 